\title{
ZnO nanorod arrays fabrication via chemical bath deposition: Ligand concentration effect study
}

\author{
Maria Elena Fragalà $^{\mathrm{a}, *}$, Yana Aleeva ${ }^{\mathrm{b}}$, Graziella Malandrino ${ }^{\mathrm{a}}$ \\ a Dipartimento di Scienze Chimiche, Università di Catania, Viale Andrea Doria 6, 95100 Catania, Italy \\ b Scuola Superiore di Catania, Università di Catania, Viale Andrea Doria 6, 95100 Catania, Italy
}

\section{A R T I C L E I N F O}

\section{Article history:}

Received 7 May 2010

Received in revised form

21 July 2010

Accepted 23 July 2010

Available online 12 August 2010

\section{Keywords:}

$\mathrm{ZnO}$

CBD

Nanorods

Ligand

Solution growth

\begin{abstract}
A B S T R A C T
A new ligand, $\mathrm{N}, \mathrm{N}, \mathrm{N}^{\prime}, \mathrm{N}^{\prime}$-tetramethylethylenediamine, has been used to grow $\mathrm{ZnO}$ nanorods on silicon substrates via a two steps approach. A preliminary seeding on silicon substrates has been combined with chemical bath deposition using a Zinc acetate - $\mathrm{N}, \mathrm{N}, \mathrm{N}^{\prime}, \mathrm{N}^{\prime}$-tetramethylethylenediamine aqueous solution. The used diamino ligand has been selected as $\mathrm{Zn}^{2+}$ complexing agent and the related hydrolysis generates the reacting ions $\left(\mathrm{Zn}^{2+}\right.$ and $\mathrm{OH}^{-}$) responsible for the $\mathrm{ZnO}$ growth. The seed layer has been annealed at low temperature $\left(<200{ }^{\circ} \mathrm{C}\right)$ and the $\mathrm{ZnO}$ nanorods have been grown on this $\mathrm{ZnO}$ amorphous layer. There is experimental evidence that the ligand concentration (ranging from 5 to $50 \mathrm{mM}$ ) strongly affects the alignment of $\mathrm{ZnO}$ nanorods on the substrate, their lateral dimension and the related surface density. Length and diameter of $\mathrm{ZnO}$ nanorods increase upon increasing the ligand concentration, while the nanorod density decreases. Even more important, it has been demonstrated, as proof of concept, that chemical bath deposition can be usefully combined with colloidal lithography for selective ZnO nanorod deposition. Thus, by patterning the $\mathrm{ZnO}$ seeded substrate with polystyrene microsphere colloidal lithography, regular Si hole arrays, spatially defined by hexagonal $\mathrm{ZnO}$ nanorods, have been successfully obtained.
\end{abstract}

(c) 2010 Elsevier Ltd. All rights reserved.

\section{Introduction}

The fabrication of nanostructures by effective, versatile and low temperature synthetic approaches capable of a systematic control of size, morphology and surface density of these fundamental nanobuilding blocks is, no doubt, of relevance nowadays.

\footnotetext{
* Corresponding author. Tel.: +39095 7385126; fax: +39 095580138.

E-mail address: me.fragala@unict.it (M.E. Fragalà).
} 
ZnO nanomaterials, with large surface to volume ratio, are particularly attractive nanosystems with promising applications in dye-sensitized solar cells, hybrid inorganic/organic multifunctional materials, chemical sensors, transparent conductors and light-emitting diodes. In fact, they have intrinsic semiconducting, optical, ferroelectric, piezoelectric, chemical and biochemical properties suited for a wide range of novel applications [1,2].

In this context, among the synthetic approaches used to fabricate well defined $\mathrm{ZnO}$ 1D nanostructures [3-7], chemical bath deposition (CBD) [8,9] certainly benefits of low temperature requirements and of simple and low-cost equipment in environmentally safe synthetic conditions. Even more important, CBD favours an accurate control of the morphology and the orientation of ZnO nanostructures by tuning the process parameters through the relevant equilibria that are established in the solution [10]. Although various shapes of $\mathrm{ZnO}$ are obtained via CBD [11,12], the systematic control of crystal shape still remains a challenge.

Hexamethylenetetramine (HMT) is one of the most used ligand in ZnO CBD process [10,13,14]. ZnO nanorod formation is promoted by HMT thermal decomposition, that provides hydroxide ions $\left(\mathrm{OH}^{-}\right)$ and ammonia molecules $\left(\mathrm{NH}_{3}\right)$ to the solution $[9,10]$, but the control of the several equilibria involved in the process is critical. Other Lewis bases, such as ethylenediamine (en) and triethanolamine (TEA), have been used in $\mathrm{ZnO} C B D$ as effective complexing agents for the $\mathrm{Zn}^{2+}$ ions and speciation calculations [10] have indicated the $\mathrm{pH}$ conditions required to form $\mathrm{ZnO}$, according to zinc hydroxide supersaturation values. Despite pH of nutrient bath is another important factor that control CBD mechanism, it has been found that $\mathrm{ZnO}$ films and nanostructures can be formed also without $\mathrm{pH}$ adjustment of bath solution. In this context, literature reports on a wide selection of $\mathrm{Zn}^{2+}$ complexing agents used in CBD $[8-10,15,16]$.

Herein, we report on the synthesis of $\mathrm{ZnO}$ nanorods via CBD using, for the first time, the $\mathrm{N}, \mathrm{N}, \mathrm{N}^{\prime}, \mathrm{N}^{\prime}$ tetramethylethylenediamine (TMEDA) ligand. This Lewis base is comparable to ethylenediamine, but its $\mathrm{Zn}^{2+}$ complexing capability is lower with respect to ethylenediamine. We scrutinized the effects of the TMEDA ligand concentration and related CBD deposition time on the $\mathrm{ZnO}$ nanostructure growth. The adopted $\mathrm{ZnO}$ nanorod $\mathrm{CBD}$ approach, requiring low temperature treatments, is suitable for integration with colloidal lithography. In this context, as a proof of concept, selective ZnO depositions have been successfully attempted and the present procedure opens up attractive challenges to produce wide varieties of $\mathrm{ZnO}$ nano-arrays.

\section{Experimental details}

ZnO nanorods (NRs) were grown on silicon substrates using a two steps approach involving a preliminary seeding of substrates. Silicon substrates (111) were etched in HF water solution (10\%) for $60 \mathrm{~s}$ in order to remove the surface native oxide, washed with water and blown with nitrogen. The $\mathrm{ZnO}$ seed layer was obtained by drop casting a $15 \mathrm{mM}$ methanolic solution of zinc acetate $\left(\mathrm{Zn}\left(\mathrm{CH}_{3} \mathrm{COO}\right)_{2} \cdot 2 \mathrm{H}_{2} \mathrm{O}\right)$ onto the substrate and drying in air. During this step, the hydrolysis of zinc acetate in aqueous solution acts as the source of $\mathrm{OH}^{-}$ions.

$$
\begin{aligned}
& \mathrm{Zn}\left(\mathrm{CH}_{3} \mathrm{COO}\right)_{2} \rightarrow 2 \mathrm{CH}_{3} \mathrm{COO}^{-}+\mathrm{Zn}^{2+} \\
& \mathrm{CH}_{3} \mathrm{COO}^{-}+\mathrm{H}_{2} \mathrm{O} \leftrightarrows \mathrm{CH}_{3} \mathrm{COOH}+\mathrm{OH}^{-} \\
& \mathrm{Zn}^{2+}+2 \mathrm{OH}^{-} \rightarrow \mathrm{Zn}(\mathrm{OH})_{2}
\end{aligned}
$$

The procedure was repeated twice to allow a uniform coverage of the substrate. The $\mathrm{ZnO}$ seed layer was afterwards annealed in a tubular reactor at $150^{\circ} \mathrm{C}$ for $5 \mathrm{~min}$ to remove solvents and contaminants. Note that, the applied thermal treatment is not enough to produce a crystalline layer. Finally, $\mathrm{ZnO}$ nanostructures were grown on these seeded substrates by chemical bath deposition (CBD) method.

The zinc acetate $\left(\mathrm{Zn}\left(\mathrm{CH}_{3} \mathrm{COO}\right)_{2} \cdot 2 \mathrm{H}_{2} \mathrm{O}\right)$ dissolved in distilled water at $70{ }^{\circ} \mathrm{C}$ was admixed with a separately prepared $\mathrm{N}, \mathrm{N}, \mathrm{N}^{\prime}, \mathrm{N}^{\prime}$-tetramethylethylenediamine (TMEDA) solution in water. The zinc acetate and the TMEDA ligand concentrations were kept to a 1:1 molar ratio in all the experiments (in order to maintain the same degree of supersaturation), while the molar concentrations were ranging from 5 to $50 \mathrm{mM}$. No pH adjustment has been done. The resulting cloudy ZnO-containing suspensions were maintained at $70{ }^{\circ} \mathrm{C}$ without stirring for a time ranging from 2 to $4 \mathrm{~h}$ to grow $\mathrm{ZnO}$ nanorods and 
to investigate, in addition, the effect of deposition time. The substrates were immersed vertically in the solution. At the end of the process all the samples were sonicated in methanol for $15 \mathrm{~min}$, washed with distilled water and dried in nitrogen ambient.

Selective $\mathrm{ZnO}$ depositions were successfully attempted using colloidal lithography. Monodispersed polystyrene microspheres have been arranged on silicon substrates by dewetting-driven selfassembly. ZnO seed layer has been deposited by drop casting on patterned substrates and annealed at low temperature $\left(350{ }^{\circ} \mathrm{C}\right)$. The obtained hybrid organic-inorganic (PS-ZnO) system was used as template for $\mathrm{ZnO}$ nanorod growth via CBD process.

Morphology of obtained $\mathrm{ZnO}$ nanorods have been examined by field emission scanning electron microscopy (FE-SEM) using a ZEISS VP 55 microscope. $\theta-2 \theta$ X-ray diffraction (XRD) patterns were recorded with a Bruker-AXS D5005 $\theta-\theta$ X-ray diffractometer, using $\mathrm{Cu} \mathrm{K}_{\alpha}$ radiation operating at $40 \mathrm{kV}$ and $30 \mathrm{~mA}$.

\section{Results and discussion}

Zinc acetate has been used as precursor to deposit, by drop casting, ZnO seed layer on silicon substrates. ZnO nanostructures have been grown on these seeded Si substrates by chemical bath deposition method (CBD).

Diamino-based ligands are often used in CBD due to the dual ability to complex zinc cations $\left(\mathrm{Zn}^{2+}\right)$ and undergo basic hydrolysis. As a matter of choice the $\mathrm{N}, \mathrm{N}, \mathrm{N}^{\prime}, \mathrm{N}^{\prime}$-tetramethylethylenediamine, often used in the metal-organic precursor synthesis [17] and related MOCVD deposition [18] can be considered as an effective ligand for $\mathrm{Zn}^{2+}$ (Eq. (1)) and the hydrolytic equilibrium of the related complex controls the overall CBD growth, by a gradual release of $\mathrm{Zn}^{2+}$ and $\mathrm{OH}^{-}$reactants.

$$
\begin{aligned}
& {[\mathrm{Zn}(\mathrm{TMEDA})]^{2+} \leftrightarrows \mathrm{Zn}^{2+}+\text { TMEDA }} \\
& \text { TMEDA }+\mathrm{H}_{2} \mathrm{O} \leftrightarrows \mathrm{TMEDA}^{-\mathrm{H}^{+}}+\mathrm{OH}^{-} \\
& \mathrm{Zn}^{2+}+2 \mathrm{OH}^{-} \rightarrow \mathrm{Zn}(\mathrm{OH})_{2} \downarrow \rightarrow \mathrm{ZnO} \downarrow+\mathrm{H}_{2} \mathrm{O}
\end{aligned}
$$

At the experimental temperature value $\left(70{ }^{\circ} \mathrm{C}\right.$ in the present case) the $\mathrm{Zn}$-TMEDA complex equilibrium is, in fact, perturbed and the diamino-ligand undergoes basic hydrolysis.

Several authors $[8-10,19]$ have reported on the buffer effect played by the amino-based ligand during the CBD. No doubt, in fact, that equilibria 1 and 2 regulate the concentration of the $\mathrm{Zn}^{2+}$ and $\mathrm{OH}^{-}$ions in the solution. Thus, when their ionic product (IP) exceeds the $\mathrm{Zn}(\mathrm{OH})_{2}$ solubility product constant $\left(\mathrm{K}_{\mathrm{ps}}\right)$ the precipitation of $\mathrm{ZnO}$ nuclei becomes activated, due to the presence of free $\mathrm{Zn}^{2+}$ and $\mathrm{OH}^{-}$ions. $\mathrm{Zn}(\mathrm{OH})_{2}$ and $\mathrm{ZnO}$ are thus formed in the aqueous solution. The precipitates of $\mathrm{Zn}(\mathrm{OH})_{2}$ are more soluble, compared to the $\mathrm{ZnO}$ precipitates, and therefore, they represent a reservoir of $\mathrm{Zn}^{2+}$ and $\mathrm{OH}^{-}$ions, which, in turn, form the $\mathrm{ZnO}$ nuclei that represent the building blocks for the formation of the nanorods.

The variation of the concentration of the complexing agent, of course, modifies the $\mathrm{OH}^{-}$ concentration and, hence, enhances the film growth rate. On the other hand, it increases the solution supersaturation level [20] (which promotes an undesirable bulk precipitation), thus leading to the reduction of the film growth. In particular, it is known that in $\mathrm{ZnO} C B D$, the homogeneous growth mechanism is promoted by the deposition of $\mathrm{ZnO}$ clusters formed in the solution [21]. It competes with the heterogeneous mechanism that favours the deposition at the solid/solution interface [22-24]. The heterogeneous process prevails during the initial step of CBD while, upon increasing the growth time, the homogeneous growth acts as a stopping process, thus inhibiting the further growth of nanostructures. In fact, at the end of the CBD process, the grown $\mathrm{ZnO}$ nanostructures present bimodal morphology consisting of an underlying nanorod arrangement covered with porous and poorly adherent, microstructure agglomeration (Fig. 1). Despite the poor adherence, rinsing with distilled water is not enough to remove from the substrates the $\mathrm{ZnO}$ agglomerates homogeneously deposited. Nevertheless, sonication in methanol has proven effective to obtain clean and well adherent nanorod arrays.

In this general context, a successful strategy has been presently developed and it has proven capable of growing, on seeded silicon substrates, $\mathrm{ZnO}$ nanorods, having a diameter ranging from 


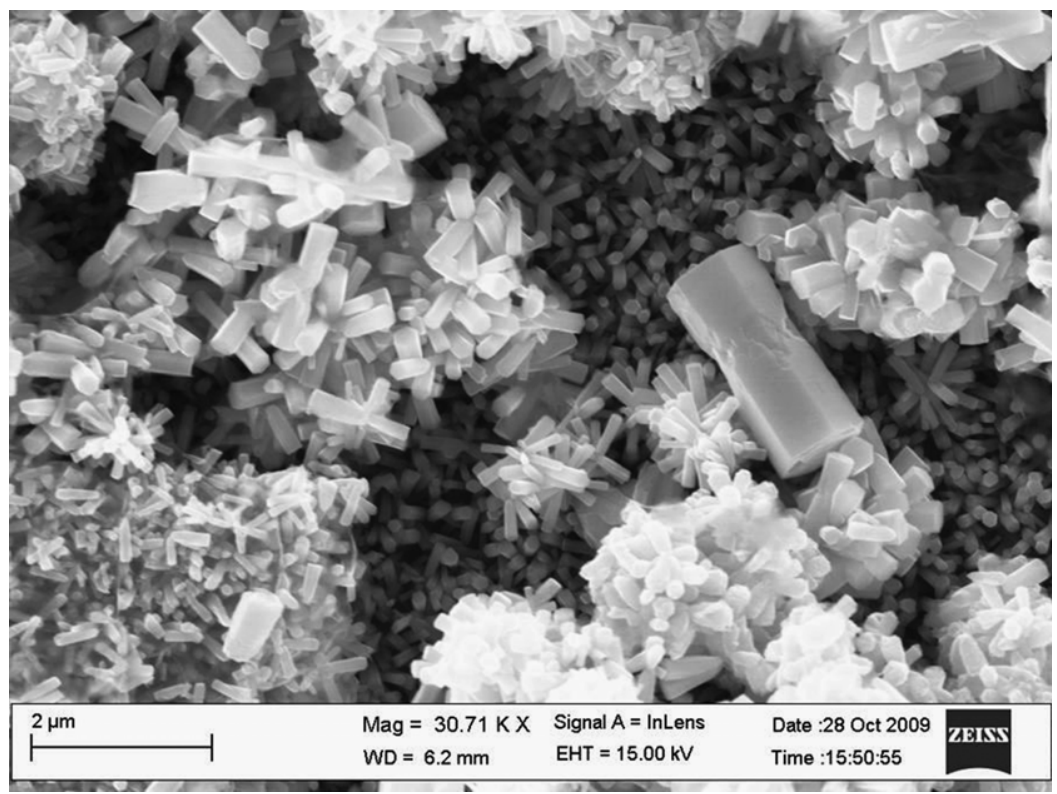

Fig. 1. $\mathrm{ZnO}$ nanostructures deposited on $\mathrm{ZnO}$ seeded substrates at the end of the CBD process, before washing steps.

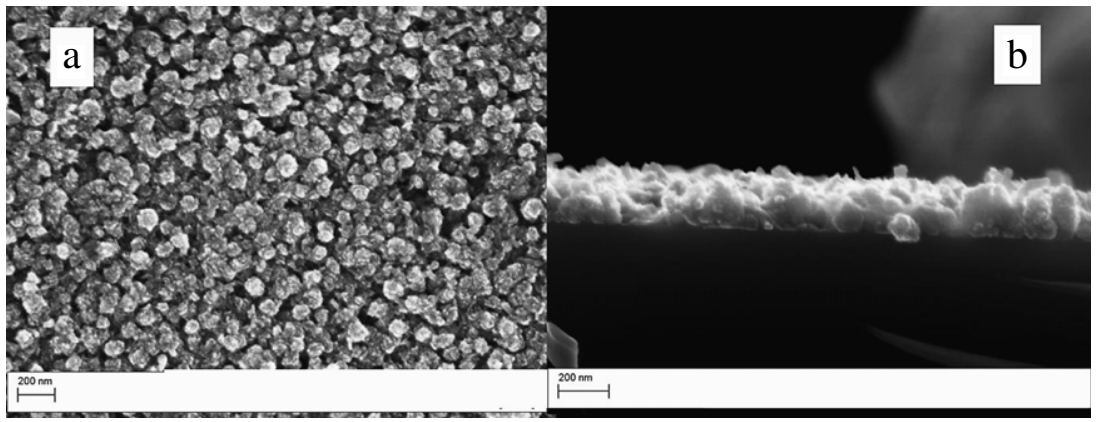

Fig. 2. ZnO nanorods obtained after $2 \mathrm{~h}$ CBD using TMEDA 5mM.

100 to $500 \mathrm{~nm}$, with a perfect hexagonal shape throughout their lengths and preferentially oriented along the $c$-axis direction. Particular attention has been devoted to study the effect of TMEDA ligand concentration on $\mathrm{CBD}$ of $\mathrm{ZnO}$ nanorods and to define clean and reproducible procedures to obtain nanostructures having tailored density and dimensions. There is evidence that the ligand concentration affects both the length and the width of the $\mathrm{ZnO}$ nanorods, thus resulting in a different nanostructure density.

Lower TMEDA concentration $(5 \mathrm{mM})$ and shorter $(2 \mathrm{~h})$ CBD process time produce a $\mathrm{ZnO}$ nanostructured layer having a granular structure (Fig. 2(a)). The SEM cross section image indicates a mean film thickness of $200 \mathrm{~nm}$ with an average grain dimension of $\sim 100 \mathrm{~nm}$ (Fig. 2(b)). As a matter of fact, $\mathrm{ZnO}$ nanograins evolve to nanorods and their lengths and diameters strongly depend upon the chosen TMEDA concentration. In particular, the $15 \mathrm{mM}$ concentration $(2 \mathrm{~h})$ results in $\mathrm{ZnO}$ nanorods having diameter of $\sim 80 \mathrm{~nm}$ (Fig. 3(a)) and length of $\sim 800 \mathrm{~nm}$ (Fig. 3(b)). Growth time is an additional factor that affects the nanorod density and their final length. Thus, increasing the growth time to $4 \mathrm{~h}$, the nanostructures appear less denser compared to shorter deposition time $(2 \mathrm{~h})$ counterparts. In addition, the overall diameter increases from $\sim 80$ to $\sim 150 \mathrm{~nm}$ (Fig. 3(c)). 

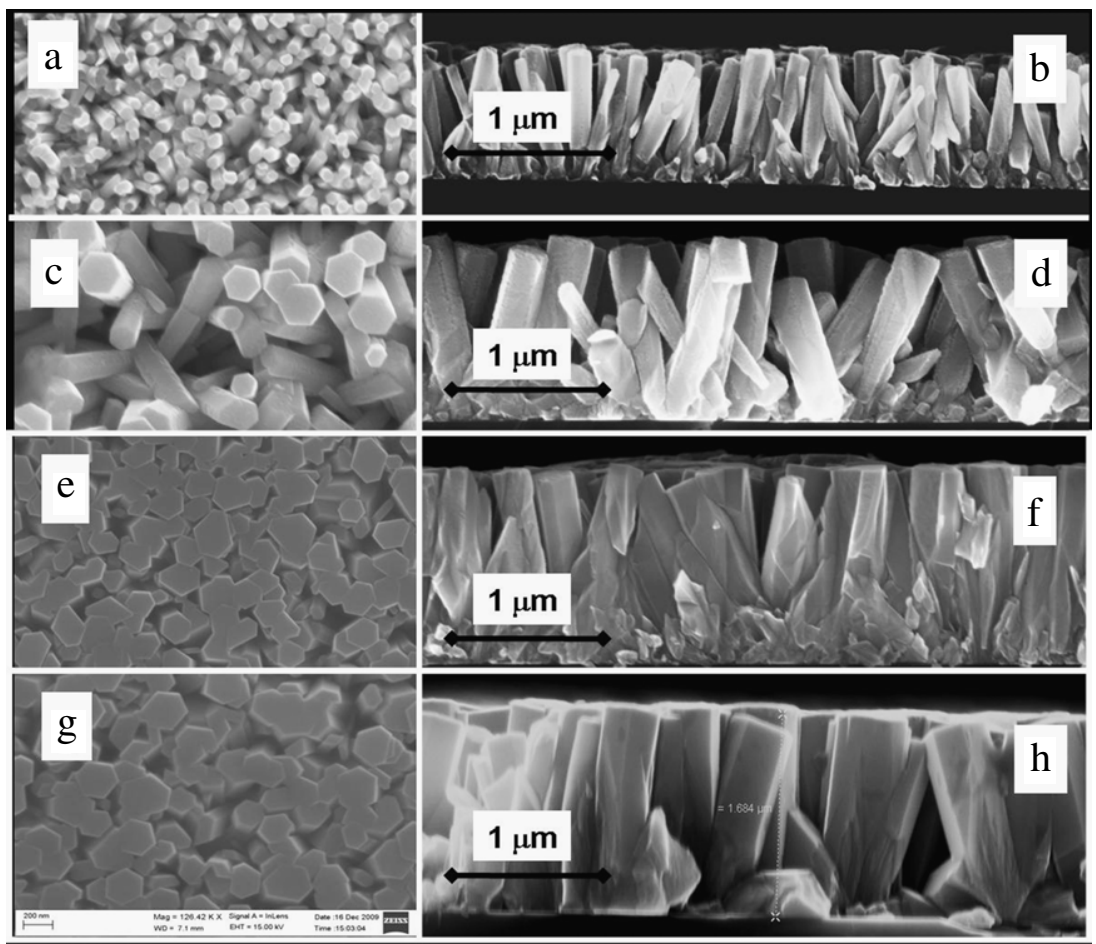

Fig. 3. Plan view (a) and cross section view (b) of ZnO nanorods obtained after $2 \mathrm{~h} \mathrm{CBD}$ using TMEDA $15 \mathrm{mM}$; Plan view (c) and cross section view (d) of ZnO nanorods obtained after $4 \mathrm{~h}$ CBD using TMEDA $15 \mathrm{mM}$; Plan view (e) and cross section view (f) of ZnO nanorods obtained after 2 h CBD using TMEDA 50 mM; Plan view (g) and cross section view (h) of ZnO nanorods obtained after $4 \mathrm{~h}$ CBD using TMEDA $50 \mathrm{mM}$.

It is evident from the SEM images, that the top surface of these nanorods appears smooth and hexagonally shaped whilst the lateral sides look porous and not yet geometrically well defined (Fig. 3(d)). SEM data also point to an increased nanorod length up to $1.4 \mu \mathrm{m}$ (Fig. 3(d)), unfortunately associated with the out-of-vertical alignment. The overall nanorod dimensions obtained using the $15 \mathrm{mM}$ concentration are smaller than the structures grown using a $50 \mathrm{mM}$ concentration $(2 \mathrm{~h})$, having a diameter of $\sim 200 \mathrm{~nm}$ (Fig. 3(e)). Moreover, the nanorod length increases from $800 \mathrm{~nm}$ to $1.4 \mu \mathrm{m}$ (Fig. 3(f)) upon increasing TMEDA concentration from 15 to $50 \mathrm{mM}$ (using $2 \mathrm{~h}$ as growth time). Even more interesting, better oriented and hexagonally shaped nanostructures are obtained at $50 \mathrm{mM}$, whilst lower values $(15 \mathrm{mM})$ produce less dense nanorods being slant away from the vertical direction. Longer growth times $(4 \mathrm{~h})$ associated with higher TMEDA concentration (50 mM) cause the densification of the nanowires in accordance with the disappearance of the pores and the formation of very smooth and compact nanostructures (Fig. $3(\mathrm{~g})$ ). Interesting enough there is also evidence of some coalescence (from their bottom to the top) (Fig. 3(h)), as the consequence of a diameter expansion due to a preferential longitudinal growth compared with the $c$-texturing. This latter effect can be accounted for by considering the different growth rates associated with the different family of planes $[25,26]$ of the wurtzite crystal structure. In particular, the faster growth is observed for the polar family [1] followed by [01-1-1] $>[01-10]>[01-11]>[000-1]$ [27]. It, therefore, transpires that $\mathrm{ZnO}$ deposition associated with CBD involves a preferential mass transport to particular crystal directions in the existing structures, thus favouring vertically aligned $\mathrm{ZnO}$ nanorods. This observation finds counterpart in the XRD pattern (Fig. 4) that shows the prevailing intensity of (002) peak as an evidence of the $c$-axis texturing vertical to the substrate. In particular, at the lowest concentration $(5 \mathrm{mM})$, the typical wurtzite diffraction pattern is slightly visible, while upon increasing the concentration, the crystallinity improves and the (002) orientation becomes 
a

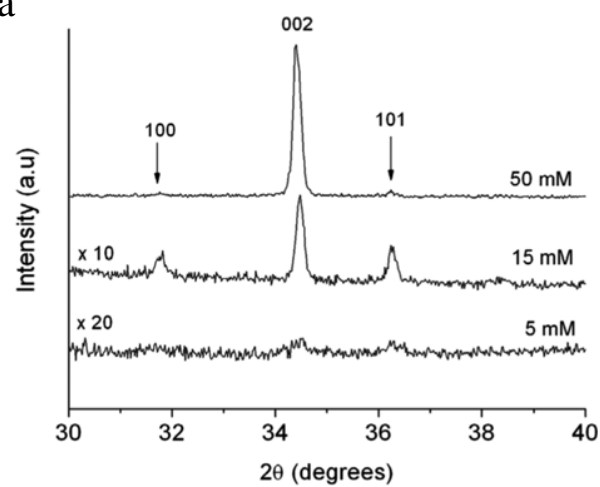

b

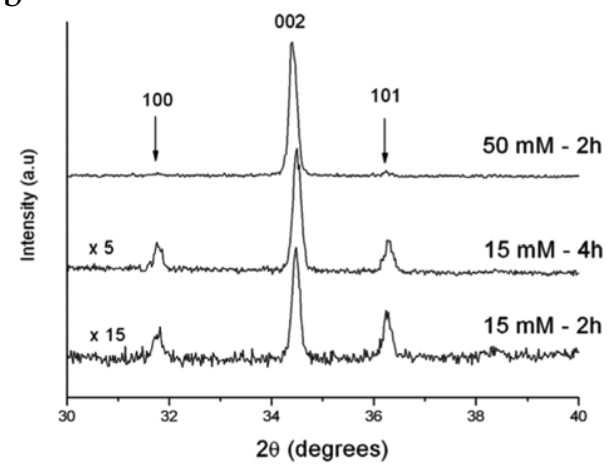

Fig. 4. XRD patterns of $\mathrm{ZnO}$ nanorods ( $2 \mathrm{~h}$ CBD) obtained using different TMEDA concentrations (a) and relative peak intensity comparison by varying CBD time and concentration (b).

dominant (Fig. 4(a)). Fig. 4(b) shows the evolution of the crystallographic preferential orientation along the $c$-axis, upon increasing TMEDA concentration and CBD deposition time $(2 \mathrm{~h} \mathrm{vs} 4 \mathrm{~h})$. At $15 \mathrm{mM}$ concentration, the SEM evidences are well confirmed by XRD data showing the increasing predominance of the (002) diffraction peak with respect to the (100) and (101) ones. At higher TMEDA concentration $(50 \mathrm{mM}, 2 \mathrm{~h}$ ), the $\mathrm{ZnO}$ nanorods are highly $c$-axis oriented. Nevertheless, both the better defined hexagonal geometry and the sidewall compactness point to some lateral mass uptake that competes with the top vertical elongation, after an induction period.

Therefore, we can conclude that $50 \mathrm{mM}$ TMEDA concentration is effective to grow 1D $\mathrm{ZnO}$ crystalline nanorods, while lower concentrations result in more slanted and polycrystalline nanostructures. This finding represents a safe evidence of a more selective nanorod growth along the $c$-axis driven by the higher ligand concentration in the nutrient solution [27].

Finally, it is worthy to note that the proposed CBD process can be totally integrated with colloidal lithography [28] and, hence, with related nanopatterned substrates. In fact, it is of particular importance the development of a fabrication strategy to grow $1 \mathrm{D} \mathrm{ZnO}$ nanostructure arrays employing low temperature aqueous solution methods.

It has been already reported about the fabrication of patterned $1 \mathrm{D} \mathrm{ZnO}$ nanostructures, directly on zinc foils [29]. Herein, as a proof of concepts, it has been presently realized a successful selective CBD ZnO deposition on silicon substrates patterned by colloidal lithography. Spherical polystyrene (PS) colloidal particles of $1 \mu \mathrm{m}$ of diameter are immobilized onto silicon surface by dewetting-driven self-assembling from aqueous dispersion. At the end of the process the colloids are close packed into two-dimensional crystal lattices and the nearly monodisperse PS microspheres ( $1 \mu \mathrm{m})$ are used to spatially define the $\mathrm{ZnO}$ nanorods, thus delimiting Si holes.

In fact, the two-dimensional (2D) arrays of colloidal microspheres act as masks for the $\mathrm{ZnO}$ seed layer deposited by drop casting through the interstices between the PS spheres. After the seeding, thermal treatments in $\mathrm{O}_{2}$ atmosphere are used to calcinate the PS microsphere. The subsequent CBD process, performed on the patterned $\mathrm{ZnO}$ seed layer, realizes an ordered array of Si holes spatially confined by $\mathrm{ZnO}$ nanorods. The $\mathrm{ZnO}$ nanorods grown on unseeded regions, previously covered by PS spheres, are finally mechanically removed (sonication) and a significant extent of patterned nanorod arrays is achieved (Fig. 5). It is interesting to note how a bimodal ZnO nanorod growth can be clearly observed as result of a local nuclei concentration gradient created in the different regions of the patterned substrate. In fact, in the substrate region not covered by PS spheres, the ZnO nanorods grow on a homogeneous $\mathrm{ZnO}$ seed layer and their diameters are clearly larger $(\sim 200 \mathrm{~nm})$ than the dimensions $(\sim 80 \mathrm{~nm})$ observed in the patterned regions, just within the PS spheres (see the inset of Fig. 5). This effect can be explained by assuming that in the patterned areas of the substrate the local concentration of $\mathrm{ZnO}$ nuclei is lower than that obtained in the unpatterned region. The resulting $\mathrm{ZnO}$ growth is, therefore, in good agreement with the ligand concentration effect observed 


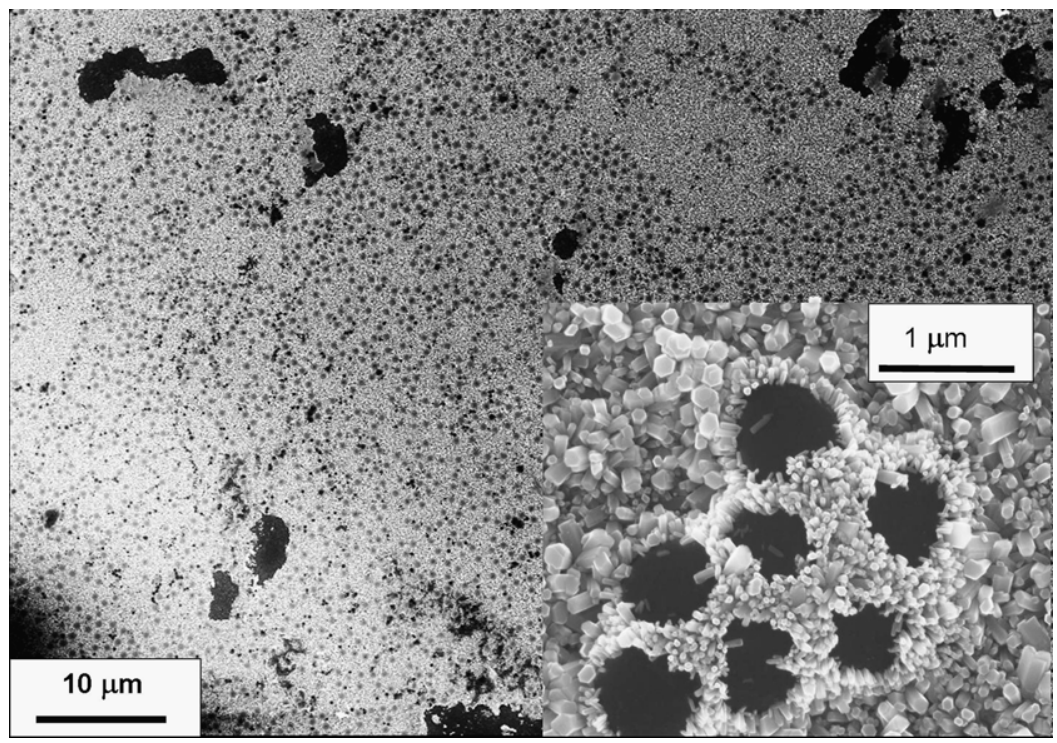

Fig. 5. Si microholes confined by ZnO nanorods obtained by patterning the seeded substrate by colloidal lithography. Bimodal morphology of $\mathrm{ZnO}$ nanorods is well visible between the patterned and unpatterned regions (inset).

on homogeneous substrates, i.e. lower concentrations produces smaller and slanted nanorods than the ones formed using higher concentration values.

\section{Conclusions}

ZnO nanorods have been fabricated adopting CBD deposition in a two steps approach, using a new amino ligand (TMEDA). Despite the amorphous nature of the $\mathrm{ZnO}$ seed layer (low postannealing temperature $<200{ }^{\circ} \mathrm{C}$ has been used), crystalline and $c$-axis oriented $\mathrm{ZnO}$ nanorods have been successfully attained. The effect of ligand concentration on ZnO CBD has been studied at different growth time, to find the best conditions to obtain discrete and long $\mathrm{ZnO}$ nanorods. There is evidence that, upon varying the TMEDA concentration, the resultant modulation of $\mathrm{Zn}^{2+}$ and $\mathrm{OH}^{-}$ion concentrations in the nutrient bath drives the formation of nanorods having different surface densities. In particular, lower TMEDA concentration produces a nanorod density higher than that obtained upon increasing the concentration values. In fact, higher concentrations promote the formation of larger nanorods, due to the coalescence of nanostructures, but promote the growth along the $c$-axis, leading to more crystalline and oriented $\mathrm{ZnO}$ nanorods. Moreover, shorter growth time reduces the nanorod density at any concentration, thus resulting in an additional tuning parameter.

The controlled ZnO CBD growth, attained using for the first time the TMEDA ligand, has been well integrated with colloidal lithography and regular Si microhole arrays, spatially defined by hexagonal $\mathrm{ZnO}$ nanorods, have been successfully obtained.

To conclude, the present process is simple, reproducible, low-cost, and poorly hazardous and even more relevant; it proves effective for an integrated approach with colloidal lithography, in order to build up high surface area nanoplatforms suitable for sensing and photo-catalytic applications.

\section{References}

[1] Z.L. Wang, Mater. Sci. Eng., R. 64 (2009) 33-71.

[2] C.D. Lokhande, P.M. Gondkar, Rajaram. S. Mane, V.R. Shinde, Sung-Hwan Han, J. Alloys Compd. 475 (2009) 304-311.

[3] C. Vargas-Hernandez, F.N. Jimenez-Garcia, J.F. Jurado, V. HenaoGranada, Microelectron. J. 39 (2008) 1349-1350.

[4] J. Li, S. Srinivasan, G.N. He, J.Y. Kang, S.T. Wu, F.A. Ponce, J. Cryst. Growth. 310 (2008) 599-603.

[5] S. O’Brien, L.H.K. Koh, G.M. Crean, Thin Solid Films. 516 (2008) 1391-1395. 
[6] S. Baruah, J. Dutta. Sci. Technol. Adv. Mater. 10 (2009) 013001.

[7] T.A. Vijayan, R. Chandramohan, S. Valanarasu, J. Thirumalai, S. Venkateswaran, T. Mahalingam, S.R. Srikumar, Sci. Technol. Adv. Mater. 9 (2008) 035007.

[8] Sung-Hak Yi, Seung-Kyu Choi, Jae-Min Jang, Jung-A Kim, Woo-Gwang, J. Colloid Interface Sci. 313 (2007) 705-710.

[9] L.L. Yang, Q.X. Zhao, Magnus Willander, J. Alloys Compd. 469 (2009) 623-629.

[10] K. Govender, D.S. Boyle, P.B. Kenway, P. O’Brien, J. Mater. Chem. 14 (2004) 2575-2591.

[11] H. Zhang, J. Feng, J. Wang, M. Zhang, Mater. Lett. 61 (2007) 5202-5205.

[12] X. Hu, Y. Masuda, T. Ohji, K. Kato, J. Am. Ceram. Soc. 92 (2009) 922-926.

[13] G. Kenanakis, D. Vernardou, E. Koudoumas, N. Katsarakis, J. Crystal Growth 311 (2009) 4799-4804.

[14] Y. Tao, M. Fu, A. Zhao, D. He, Y. Wang, J. Alloys Comp. 489 (2010) 99-102.

[15] J. Zhao, Z.-G. Jin, T. Li, X.-X. Liu, J. Eur. Ceram. Soc. 26 (2006) 2769-2775.

[16] R. Saravana Kumara, P. Sudhagarb, R. Sathyamoorthya, P. Matheswarana, Y.S. Kang, Superlattices Microstruct. 46 (6) (2009) 917-924.

[17] G. Malandrino, M. Blandino, L.M.S. Perdicaro, I.L. Fragalà, P. Rossi, P. Dapporto, Inorg. Chem. 44 (2005) 9684-9689.

[18] G. Malandrino, M. Blandino, M.E. Fragalà, M. Losurdo, G. Bruno, J. Phys. Chem. 112 (2008) 9595-9599.

[19] Q. Li, J. Bian, J. Sun, J. Wang, Y. Luo, K. Sun, D. Yu, Appl. Surf. Sci. 256 (2010) 1698-1702.

[20] M. Kostoglou, N. Andritsos, A.J. Karabelas, Ind. Eng. Chem. Res. 39 (2000) 3272-3283.

[21] Y. Masuda, K. Kato, Cryst. Growth Des. 9 (2009) 3083-3088.

[22] X. Liu, Z. Jin, S. Bu, J. Zhao, Z. Liu, Mater. Lett. 59 (2005) 3994-3999.

[23] M. Ortega-López, A. Avila-García, M.L. Albor-Aguilera, V.M. Sánchez Resendiz, Mater. Res. Bull. 38 (2003) 1241-1248.

[24] Z. Yang, Y.-Y. Shi, X.-L. Sun, H.-T. Cao, H.-M. Lu, X.-D. Liu, Mater. Res. Bull. 45 (2010) 474-480.

[25] W.J. Li, E.W. Shi, W.Z. Zhong, Z.W. Yin, J. Cryst. Growth. 203 (1999) 186-196.

[26] R.A. Laudise, A.A. Ballman, J. Phys. Chem. 64 (1960) 688-691.

[27] H.Q. Le, S.J. Chua, Y.W. Koh, K.P. Loh, E.A. Fitzgerald, J. Cryst. Growth. 293 (2006) 36-42.

[28] M.E. Fragalà, C. Satriano, G. Malandrino, Chem. Comm. (2009) 839-841.

[29] C. Li, G. Hong, P. Wang, D. Yu, L. Qi, Chem. Mater. 21 (2009) 891-897. 